Notre Dame Journal of Formal Logic Volume XVII, Number 3, July 1976 NDJFAM

\title{
TWO NOTES ON ACKERMANN'S SET THEORY
}

\author{
JOHN LAKE
}

We give solutions to two problems which concern Ackermann's set theory, A.* This theory was introduced in [1] and it is now formulated in the first-order predicate calculus with identity, using $\epsilon$ for membership and an individual constant, $\vee$, for the class of all sets. We use the letters $\phi, \Psi$, $\theta$, and $\chi$ to stand for formulae which do not contain $V$ and capital Greek letters to stand for any formulae. Then the axioms of $A$ are the universal closures of

$$
\begin{aligned}
& A 1 \quad \forall t(t \in x \leftrightarrow t \in y) \rightarrow x=y, \\
& A 2 \quad \exists z \forall t(t \in z \leftrightarrow t \in \vee \wedge \Theta), \\
& A 3 \quad x \in \vee \wedge(t \in x \vee \forall u(u \in t \rightarrow u \in x)) \rightarrow t \in \vee, \\
& A 4 \quad x, y \in \vee \wedge \forall t(\Psi(x, y, t) \rightarrow t \in \vee) \rightarrow \exists z \in \vee \forall t(t \in z \leftrightarrow \Psi(x, y, t)),
\end{aligned}
$$

where all free variables are shown in $A 4$ and $z$ does not occur in the $\Theta$ of A2. $\mathrm{A} *$ is $\mathrm{A}$ augmented by the axiom

A5 $x \in \vee \wedge \exists u u \in X \rightarrow \exists u \in x \forall t \in u t \notin x$.

Firstly, we shall solve a problem from [3], by extending some of the work on permutation models (see [2], for instance) to models of $\mathbf{A}$.

Definition 1 A functional formula $y=F(x)$ is said to be a permutation if it represents a bijection of the universe onto itself. If $y=F(x)$ is a permutation then we write $x \epsilon_{F} y$ for $F(x) \in y$ and $\Psi_{F}$ for the formula $\Psi$ with all instances of $\epsilon$ replaced by $\epsilon_{F}$.

Theorem 2 If $y=F(x)$ is a functional $\epsilon$-formula such that

(i) $F$ is a permutation,

(ii) $x \in \vee$ iff $F(x) \in \vee$,

then we can interpret $\mathrm{A}$ in $\mathrm{A}$ using $\epsilon_{\mathrm{F}}$ for the membership relation and $\vee$ for $\mathrm{V}$.

\footnotetext{
*The author acknowledges the support of the Science Research Council.
} 
Proof: Firstly, note that $x \epsilon_{F} \vee \leftrightarrow F(x) \epsilon \vee \leftrightarrow x \epsilon \vee$, and we often use this in showing that the interpretations of the axioms hold.

$(A 1)_{F}$ Extensionality holds as $F$ is a bijection.

$(A 2)_{F}$ We show that $\exists z \forall t\left(t \epsilon_{F} z \leftrightarrow t \epsilon \vee \wedge \Psi_{F}\right)$. By $A 2$ and (ii) $\exists z \forall p(p \epsilon$ $\left.z \leftrightarrow \exists t \epsilon \vee\left(p=F(t) \wedge \Psi_{F}\right)\right)$. Then $t \epsilon_{F} z \leftrightarrow F(t) \epsilon z \leftrightarrow t \epsilon \vee \wedge \Psi_{F}$, as required.

$(A 3)_{F}$ We firstly show that $x \epsilon_{F} y \in \vee \rightarrow x \in \vee . x \epsilon_{F} y \rightarrow F(x) \in y \rightarrow F(x) \in \vee \rightarrow$ $x \in \mathrm{V}$ by $A 3$ and (ii). Now it remains to show that

$$
\forall t\left(t \epsilon_{F} x \rightarrow t \epsilon_{F} y\right) \wedge y \in \mathrm{V} \rightarrow x \in \mathrm{V} .
$$

$\forall t\left(t \epsilon_{F} x \rightarrow t \epsilon_{F} y\right) \rightarrow \forall t(F(t) \epsilon x \rightarrow F(t) \in y)$ by definition,

$\rightarrow x \subseteq y$ by (ii),

$\rightarrow x \in \mathrm{V}$ by $A 3$.

$(A 4)_{F}$ Suppose that $x, y \in \vee$ and $\forall t\left(\phi_{F}(x, y, t) \rightarrow t \in \mathrm{V}\right)$, and we show that $\exists z \in \vee \forall t\left(t \epsilon_{F} z \leftrightarrow \phi_{F}(x, y, t)\right)$. Let $\Psi(x, y, z)$ be the formula $\exists t(p=F(t) \wedge$ $\left.\phi_{F}(x, y, t)\right)$ and then from (ii) we get $\forall p(\Psi(x, y, p) \rightarrow p \in \mathrm{V})$. Then, by $A 4$, $\exists z \epsilon \vee \forall p\left(p \epsilon z \leftrightarrow \Psi(x, y, p)\right.$. Now, $t \epsilon_{\mathrm{F}} z \leftrightarrow F(t) \epsilon z \leftrightarrow \phi_{\mathrm{F}}(x, y, t)$, so that $(A 4)_{F}$ holds.

Q.E.D.

Question 4.24(c) of [3] asks "If we add the following schema of downward reflection to $A$, then do we get $A *$ ?

DR If $\phi$ has exactly two free variables, then $y \in \vee \wedge \phi(\vee, y) \rightarrow \exists x \in \vee \phi(x, y)$.",

We shall answer this question negatively, provided that $Z F$ is consistent. From [3], the consistency of ZF implies the consistency of $A^{*}$ and $A^{*} \vdash D R$ so that it suffices to give an interpretation of $A+D R$ in $A+D R$ in such a way that the interpretation of $A 5$ fails. We do this as follows:

Let $y=F(x)$ be a functional $\epsilon$-formula which says that

$$
\begin{aligned}
& F(72)=\{72\}, \\
& F(\{72\})=72,
\end{aligned}
$$

and

$$
F(x)=x \text {, otherwise. }
$$

$F$ obviously satisfies the hypothesis of Theorem 2 so that result shows that we can interpret $A$ in $A+D R$, using $\epsilon_{F}$ for membership. An instance of $(\mathrm{DR})_{F}$ becomes

$$
y \in \vee \wedge \phi_{F}(\vee, y) \rightarrow \exists x \in \vee \phi_{F}(x, y) .
$$

This is just another instance of DR so that we can interpret $A+D R$ in $\mathrm{A}+\mathrm{DR}$, using $\epsilon_{F}$ for membership. The interpretation of $A 5$ does not hold as $x \epsilon_{F}\{72\} \leftrightarrow F(x) \epsilon\{72\} \leftrightarrow F(x)=72 \leftrightarrow x=\{72\}$, as required.

Now we shall consider an extension of $A$ which was suggested by Wang. On page 428 of [4] he suggests that it might be possible to allow any formula to occur in $A 4$ if we modify the axiom to 
$\left.A 4_{\mathrm{w}} x, y \in \vee \wedge \forall t(\Phi(x, y, t) \rightarrow t \in \mathrm{\vee}) \wedge \exists t \in \vee\right\urcorner \Phi(x, y, t) \rightarrow$

$\exists z \in \vee \forall t(t \in z \leftrightarrow \Phi(z, u, t))$,

where all free variables are shown. He also mentions that it might be necessary to add the existence of the empty set as an axiom, but it is straightforward to check that there is a model of such axioms in which $V=\{\phi\}$. However, Theorem 3 shows that if we add an axiom asserting the existence of at least two sets, then the theory becomes inconsistent.

Theorem 3 The theory with axioms $A 1, A 2, A 3, A 4$, and $\exists x \in \vee \exists y \in \vee x \neq y$ is inconsistent.

Proof: In this theory we firstly prove

$$
\forall x \in \mathrm{V} x \notin x \text {. }
$$

Suppose that $\exists x \in \vee x \in x$ and let $\Phi(x)$ be the formula $x \in \vee \wedge x \notin x$. Then, by $A 4_{\mathrm{w}}, z=\{t \mid t \in \vee \wedge t \notin t\} \in \vee$, but $z \in z \leftrightarrow z \notin z$, so that (*) holds. From (*), we know that

$$
\mathrm{V} \notin \mathrm{V} \text {. }
$$

Let $x \in \vee$ and we can suppose that $x \neq \phi$. Using $t \neq t$ in $A 4_{\mathrm{w}}$, we see that $\phi \in \vee$. Let $\Psi_{1}(t)$ be the formula $t \in \vee \wedge t \neq \phi$, so that by $A 4_{\mathrm{w}} z^{\prime}=\{x \mid x \in \vee \wedge x \neq$ $\phi\} \in \vee$. Let $\Psi_{2}(t)$ be $t \epsilon z^{\prime} \vee t=\phi$. Then $\forall t\left(\Psi_{2}(t) \rightarrow t \epsilon \vee\right), z^{\prime} \notin z^{\prime}$ by $(*)$ and $z^{\prime} \neq \phi$ by our assumption that there are at least two sets. Hence we can use $\Psi_{2}$ in $A 4_{\mathrm{w}}$ and this gives $\left\{x \mid x \in z^{\prime} \vee x=\phi\right\} \in \mathrm{V}$, i.e., $\vee \in \mathrm{V}$, and this contradicts $(* *)$.

Q.E.D.

\section{REFERENCES}

[1] Ackermann, W., "Zur axiomatik der mengenlehre," Mathematische Annalen, vol. 131 (1956), pp. 336-345.

[2] Felgner, U., Models of ZF Set Theory, Springer-Verlag, Berlin (1971).

[3] Reinhardt, W., “Ackermann's set theory equals ZF," The Annals of Mathematical Logic, vol. 2 (1970), pp. 189-249.

[4] Wang, H., A Survey of Mathematical Logic, North-Holland, Amsterdam (1963).

The Polytechnic of the South Bank

London, England 